\title{
Thermal Resistance-Based Bounds for the Effective Conductivity of Composite Thermal Interface Materials
}

\author{
P. Karayacoubian, M. M. Yovanovich, J. R. Culham \\ Microelectronics Heat Transfer Laboratory \\ Department of Mechanical Engineering \\ University of Waterloo \\ Waterloo, Ontario, Canada N2L 3G1 \\ Email: pkarayac@mhtlab.uwaterloo.ca
}

\begin{abstract}
Thermally enhanced greases made of dispersions of small conductive particles suspended in fluidic polymers can offer significant advantages when used as a thermal interface material (TIM) in microelectronics cooling applications. The following study presents two simple theorems for establishing bounds on the effective thermal conductivity of such inhomogeneous media. An upper bound is established when isotherms are assumed perpendicular to the direction of heat flow through the material. In a similar manner, a lower bound is established when adiabats are assumed parallel to the direction of heat flow. As an example of the application of these theorems, the TIM is assumed to be composed of a cubic array of uniform spheres in a surrounding medium. In most instances, a geometric mean of the bounding solutions determined for this case gives good agreement with experimental data available in the literature. Numerical simulations of a spherical particle in a unit cube cell confirm the validity of the model. This model is not applicable to systems in which the discontinuous phase is either well-connected throughout or has settled. The potential of extending this preliminary thermal resistance-based approach to investigate other geometries and effects associated with distribution, orientation, and boundary resistance is discussed.
\end{abstract}

$\begin{array}{ll}\text { Nomenclature } \\ A & \text { cross-sectional area }\left[\mathrm{m}^{2}\right] \\ I & \text { integral } \\ K & 1-1 / \kappa \\ k & \text { thermal conductivity }[\mathrm{W} / \mathrm{m} K] \\ L & \text { distance between boundaries }[\mathrm{m}] \\ N & \text { number of particles } \\ n & \text { local normal, number of numerical and } \\ & \text { analytical values } \\ R & \text { thermal resistance, } \Delta T / Q[K / W] \\ R M S & \text { root mean square deviation } \\ T & \text { temperature }[K] \\ \Delta T & \text { temperature drop }[K] \\ V & \text { volume }\left[\mathrm{m}^{3}\right] \\ x & \text { numerical/analytical value } \\ \epsilon & \text { radius of the particle } \\ \kappa & \text { conductivity ratio, } k_{p} / k_{m} \\ \phi & \text { volume fraction, } V_{p} /\left(V_{p}+V_{m}\right)\end{array}$

0-7803-XXXX-X/06/\$20.00 (c)2006 IEEE

$\begin{array}{ll}\text { Subscripts } & \\ 1,2 & \text { regions of the cell } \\ e & \text { effective } \\ H, L & \text { high, low } \\ l b, u b & \text { lower bound, upper bound } \\ m & \text { matrix (continuous surrounding medium) } \\ p & \text { particle (discontinuous phase) }\end{array}$

\section{Introduction}

Numerous research initiatives have addressed the minimization of thermal joint resistance in microelectronic applications. Fluidic particle-laden polymers are the latest thermal interface materials (TIMs) being investigated for these applications. The conductivity of the fluid, typically an oil, is improved by including small conductive particles. A fundamental problem which remains to be addressed is how to predict the effective thermal conductivity of these materials.

Fluidic particle-laden polymers are complex systems consisting of many small particles dispersed in a continuous phase. In most cases, the details of the microstructure are not completely known. This naturally leads one to attempt to establish bounds on the effective properties given such limited sample information. The simplest bounds for effective conductivity can be established from the particle volume fraction and physical properties of the constituents alone. In this case, the upper and lower bounds are given when the materials are arranged in parallel or series with respect to heat flow [1]. These bounds, however, can be multiple orders of magnitude apart which can be problematic when trying to use the average to obtain an accurate estimate of the effective properties. More rigorous bounding techniques using statistical correlation functions have since been developed and are described by Torquato and Milton $[2,3]$.

In the present study, two simple theorems are used to establish bounds on the effective thermal conductivity of a fluidic TIM. The dispersed phase is assumed to consist of uniform spherical particles in a cubic lattice structure such that a characteristic cell can be identified. An upper bound is then established when isotherms are assumed perpendicular to the direction of heat flow through the material. In a similar manner, 
a lower bound is established when adiabats are assumed parallel to the direction of heat flow. These bounds are more effective at constraining the solution within a narrow range of possible values and are also more relevant for typical fluidic TIMs since the dispersed phase is typically a particle suspended in the medium (and not a lamina).

\section{Literature Review}

The study of the effective conductivity of heterogeneous materials has a long history dating back to Maxwell's critical work on spherical particle suspensions published more than a century ago $[4,5]$. In light of the technological importance of determining the effective properties of heterogeneous media, a vast body of literature has since evolved. Many recent approaches such as statistical analysis and numerical simulations provide further insight into effects such as alignment of the particles, particle distribution, and particle geometry.

Table 1 summarizes models developed by various researchers which appear frequently in the literature. There are others, in particular statistical bounds and percolation theory which have been entirely neglected. Many of the other models are extensions of the Maxwell equation to incorporate effects such as particle geometry (Hamilton and Crosser [6]), boundary resistance (Benveniste [7]), thin coatings of thermally different materials (Felske [8]), and multiple poles (McPhedran and McKenzie [9]). Similar extensions can be made to the Bruggeman equation.

The available analytical models do not show good agreement with experimental results of thermally enhanced greases at large volume fractions. This is problematic as these volume fractions are typically of practical interest. As a result, a large number of semianalytical models with experimentally determined parameters have been proposed. Empirical correlations, however, tend to be more useful for correlating data than they are for making accurate predictions in a general sense.

\section{Model Development}

It is assumed that the TIM is composed of $N$ identical spherical particles dispersed throughout the continuous phase and that these particles are arranged in a lattice structure. It is further assumed that the particles and the matrix material are isotropic and have constant thermal conductivities $k_{p}$ and $k_{m}$. Because $N$ is very large, a characteristic cell can be identified as the control volume subject to analysis without introducing much error. The boundary conditions of the cell are determined from symmetry (Figure 1). The four faces of the cell parallel to the direction of heat flow are adiabatic. The other two faces are isothermal

Karayacoubian et al., Thermal Resistance-Based Bounds... with the upper surface being the hotter. Heat thus enters the control volume through the top boundary and exits through the bottom boundary.

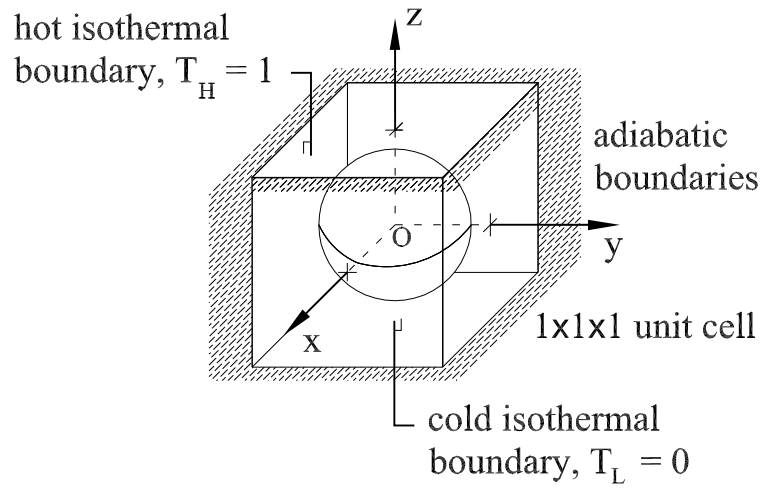

Figure 1 Characteristic (unit) cell

Steady conduction through the cell according to Fourier's law is given by

$$
Q=k_{e} A \frac{\Delta T}{L} \equiv \frac{\Delta T}{R}
$$

where $k_{e}$ is the effective thermal conductivity of the cell and therefore of the heterogeneous medium as well, $A$ is the cross-sectional area, $L$ is the distance between isothermal boundaries, $\Delta T=T_{H}-T_{L}$ is the temperature drop across the cell, and $R$ is the total resistance of the cell. Upon rearranging Equation (1),

$$
R=\frac{L}{k_{e} A}
$$

If a unit cell is selected, the substitutions $L=1 \mathrm{~m}$ and $A=L^{2}=1 m^{2}$ can be made in Equation (2), yielding

$$
k_{e}=\frac{1}{R}
$$

and if the substitution $\Delta T=1 K$ is made in Equation (1),

$$
k_{e}=Q
$$

The total thermal resistance of the cell $R$ is given by the solution of the coupled 3-D Laplace equations for the particle and the surrounding medium,

$$
\nabla^{2} T_{i}=\frac{\partial^{2} T_{i}}{\partial x^{2}}+\frac{\partial^{2} T_{i}}{\partial y^{2}}+\frac{\partial^{2} T_{i}}{\partial z^{2}}=0 \quad i=p, m
$$

The boundary conditions on the four adiabatic boundaries are given by

$$
\begin{aligned}
& \left.\left(\frac{\partial T_{m}}{\partial x}\right)\right|_{x= \pm \frac{1}{2}}=0 \\
& \left.\left(\frac{\partial T_{m}}{\partial y}\right)\right|_{y= \pm \frac{1}{2}}=0
\end{aligned}
$$

22nd IEEE SEMI-THERM Symposium 
Table 1: Effective thermal conductivity models

\begin{tabular}{|c|c|}
\hline Model & Equation \\
\hline General effective medium theory [10] & $\begin{array}{l}k_{e}=\frac{1}{4}\left(\gamma+\sqrt{\gamma^{2}+8 k_{p} k_{m}}\right) \\
\quad \text { where } \gamma=(3 \phi-1) k_{p}+[3(1-\phi)-1] k_{m}\end{array}$ \\
\hline Maxwell [4] & $k_{e}=k_{m} \frac{2 k_{m}+k_{p}-2 \phi\left(k_{m}-k_{p}\right)}{2 k_{m}+k_{p}+\phi\left(k_{m}-k_{p}\right)}$ \\
\hline Bruggeman [10] & $\left(\frac{k_{p}-k_{e}}{k_{p}-k_{m}}\right)\left(\frac{k_{m}}{k_{e}}\right)^{1 / 3}=1-\phi$ \\
\hline Woodside and Messmer [11] & $k_{e}=k_{p}^{\phi} k_{m}^{1-\phi}$ \\
\hline Cheng and Vachon [12] & $\begin{array}{l}\frac{1}{k_{e}}=\frac{1}{k_{m}}+\frac{1}{\left.\sqrt{C\left(k_{p}-k_{m}\right)\left[k_{m}+B\left(k_{p}-k_{m}\right)\right.}\right]} \times \\
\quad \ln \left[\frac{\left.\sqrt{k_{m}+B\left(k_{p}-k_{m}\right)}+B / 2 \sqrt{C\left(k_{p}-k_{m}\right.}\right)}{\left.\sqrt{k_{m}+B\left(k_{p}-k_{m}\right)}-B / 2 \sqrt{C\left(k_{p}-k_{m}\right.}\right)}\right] \\
\quad \text { where } B=\left(\frac{3 \phi}{2}\right)^{1 / 2} \text { and } C=4\left(\frac{2}{3 \phi}\right)^{1 / 2}=\frac{4}{B}\end{array}$ \\
\hline Lewis and Nielsen [13] & $\begin{array}{l}k_{e}=k_{m} \frac{1+A B \phi}{1-B C \phi} \\
\text { where } A=\{1.5,3\} \text { for }\{\text { spheres, irregular particles }\} \\
\begin{aligned} B & =\frac{k_{p} / k_{m}-1}{k_{p} / k_{m}+A} \\
C & =1+\phi\left(\frac{1-\phi_{m}}{\phi_{m}^{2}}\right), \text { and } \phi_{m}=0.637\end{aligned}\end{array}$ \\
\hline
\end{tabular}

The boundary conditions on the two isothermal boundaries are given by

$$
\begin{aligned}
T_{m}\left(x, y, \frac{1}{2}\right) & =1 \\
T_{m}\left(x, y,-\frac{1}{2}\right) & =0
\end{aligned}
$$

Perfect thermal contact between the particle and the surrounding medium is assumed. The boundary conditions at the interface are thus given by

$$
\begin{aligned}
T_{p}(x, y, z) & =T_{m}(x, y, z) \\
k_{p}\left(\frac{\partial T_{p}}{\partial n}\right) & =k_{m}\left(\frac{\partial T_{m}}{\partial n}\right)
\end{aligned}
$$

where $n$ is the local normal to the interface. In addition, the temperature at the center of the particle must remain finite, so

$$
T_{p}(0,0,0) \neq \infty
$$

The total heat flow into or out of the cell can be obtained by applying Fourier's law across either of the isothermal boundaries,

$$
Q=\left.\int_{-\frac{1}{2}}^{\frac{1}{2}} \int_{-\frac{1}{2}}^{\frac{1}{2}} k_{m}\left(\frac{\partial T_{m}}{\partial z}\right)\right|_{z=+\frac{1}{2} \text { or }-\frac{1}{2}} d x d y
$$

Equation (13) also gives the effective conductivity of the unit cell and for constant thermal conductivity, at 
the upper boundary,

$$
\frac{k_{e}}{k_{m}}=\left.\int_{-\frac{1}{2}}^{\frac{1}{2}} \int_{-\frac{1}{2}}^{\frac{1}{2}}\left(\frac{\partial T_{m}}{\partial z}\right)\right|_{z=\frac{1}{2}} d x d y
$$

The above problem is well-posed but no simple analytical solution is available. Numerical work is required to determine the temperature fields in the particle and the surrounding medium.

Two simple theorems can be used to establish bounds on the effective thermal conductivity of the cell. As an example, consider steady conduction between two infinitely long concentric cylinders. The external cylinder is heated with respect to the inner cylinder. The temperature field in the space between the two bodies is clearly 2 -D. If we allow the heat to flow directly from one point on the external cylinder to a point on the inner one and connect the various differential flux tubes in parallel, we will establish a lower bound on the heat flow between the two bodies. Conversely, if we select a differential ring in the space between the two bodies and allow heat to flow in the $\theta$-direction and then connect these multiple rings in series, we will establish an upper limit on the heat flow between the two bodies. We thus have the following two general postulates for establishing bounds on the effective thermal conductivity of a composite when conduction is the only mode of heat transfer: (1) a lower bound on the effective conductivity of our cell is established if adiabats are assumed parallel to the direction of heat flow (Figure $2(\mathrm{a})$ ) and (2) an upper bound is established if isotherms are assumed perpendicular to the direction of heat flow through the material (Figure 2 (b)).

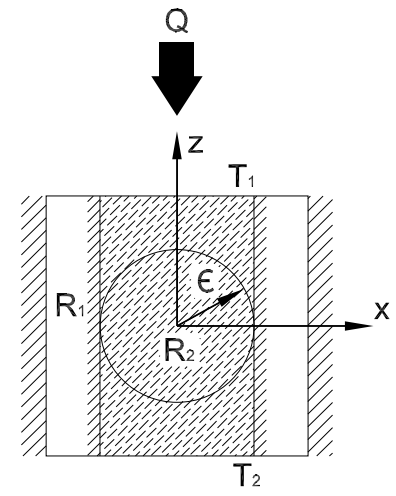

(a)

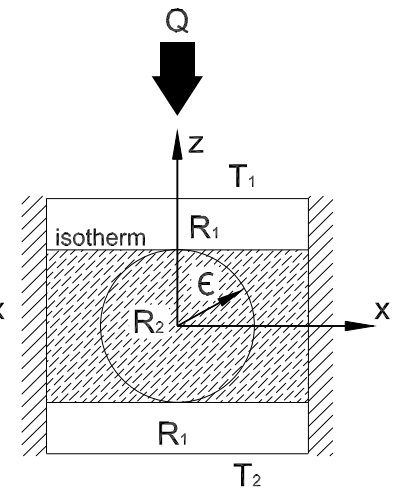

(b)
Figure 2 (a) Lower (parallel adiabats) and (b) upper (perpendicular isotherms) bounds

\section{1. $\quad$ Lower Bound - Parallel Adiabats}

When the adiabats are arranged in parallel as shown in Figure 2 (a),

$$
\frac{1}{R}=\frac{1}{R_{1}}+\frac{1}{R_{2}}=k_{e}
$$

Karayacoubian et al., Thermal Resistance-Based Bounds... where $R_{1}=\left[k_{m}\left(1-\pi \epsilon^{2}\right)\right]^{-1}$ is the resistance of the medium surrounding a right circular cylinder enclosing the particle and $R_{2}$ is resistance of the material inside the cylinder. The volume enclosed by the region inside the cylinder is symmetric so that only one quarter must be considered (Figure 3). A differential ring has two resistances in series and has a total resistance

$$
\begin{aligned}
\mathrm{d} R_{2} & =\mathrm{d} R_{2_{p}}+\mathrm{d} R_{2_{m}} \\
\text { or } \quad \frac{1}{\mathrm{~d} R_{2}} & =\frac{1}{\mathrm{~d} R_{2_{p}}+\mathrm{d} R_{2_{m}}}
\end{aligned}
$$

where $\mathrm{d} R_{2_{p}}$ and $\mathrm{d} R_{2_{m}}$ are the differential resistances of the particle and matrix in the ring. The differential resistance of the particle can be written from Fourier's law and the equation of a circle as

$$
\mathrm{d} R_{2_{p}}=\frac{z}{k_{p} \mathrm{~d} A}=\frac{\sqrt{\epsilon^{2}-x^{2}}}{k_{p} \pi x \mathrm{~d} x}
$$

where $\epsilon$ is the radius of the particle and $x$ is the radius of the ring. Likewise, the resistance of the part of the ring that is composed of the surrounding medium can be written as

$$
\mathrm{d} R_{2_{m}}=\frac{0.5-z}{k_{p} \mathrm{~d} A}=\frac{0.5-\sqrt{\epsilon^{2}-x^{2}}}{k_{p} \pi x \mathrm{~d} x}
$$

Upon combining Equations (16) to (18) and integrating,

$$
\frac{1}{R_{2}}=k_{m} 2 \pi \int_{0}^{\epsilon} \frac{x \mathrm{~d} x}{1-2 K \sqrt{\epsilon^{2}-x^{2}}}=k_{m} 2 \pi I_{l b}
$$

where

$$
\begin{gathered}
K \equiv 1-\frac{1}{\kappa} \quad \text { and } \quad \kappa \equiv \frac{k_{p}}{k_{m}} \\
I_{l b} \equiv \int_{0}^{\epsilon} \frac{x \mathrm{~d} x}{1-2 K \sqrt{\epsilon^{2}-x^{2}}}
\end{gathered}
$$

The closed form relation for the integral $I_{l b}$ is given by

$$
I_{l b}=\frac{1}{2 K^{2}} \ln \left(\frac{1}{1-2 K \epsilon}\right)-\frac{\epsilon}{K}
$$

The lower bound on the effective conductivity (nondimensionalized with the conductivity of the surrounding medium) of the cell is thus given by

$$
\left(\frac{k_{e}}{k_{m}}\right)_{l b}=1-\pi \epsilon^{2}+2 \pi I_{l b}
$$




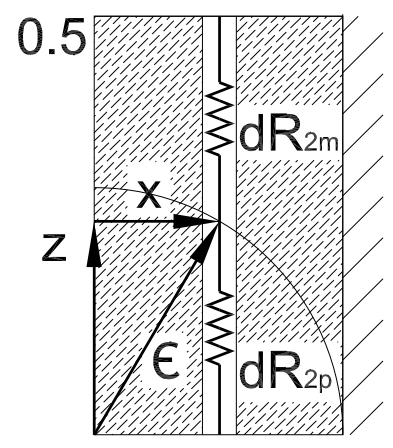

Figure $3 R_{2}$ : The thermal resistance of the material inside a right circular cylinder enclosing the particle

\subsection{Upper Bound - Perpendicular Isotherms}

When the isotherms are arranged in series as shown in Figure 2 (b),

$$
R=2 R_{1}+R_{2}=\frac{1}{k_{e}}
$$

where $R_{1}=(0.5-\epsilon) / k_{m}$ is now the resistance of the medium above and below the rectangular prism enclosing the particle. Again, we consider one quarter (half of the upper half) of the resistance of the region shaded as $R_{2}$ (Figure 4 ). A differential ring now has two resistances in parallel and has a total resistance

$$
\frac{1}{\mathrm{~d} R_{2}}=\frac{1}{\mathrm{~d} R_{2_{p}}}+\frac{1}{\mathrm{~d} R_{2_{m}}}
$$

The differential resistance of the particle can be written as

$$
\mathrm{d} R_{2_{p}}=\frac{d z}{k_{p}\left(\frac{\pi x^{2}}{2}\right)}=\frac{2 d z}{k_{p} \pi\left(\epsilon^{2}-z^{2}\right)}
$$

and that of the part of the ring composed of the surrounding medium can be written as

$$
\mathrm{d} R_{2_{m}}=\frac{d z}{k_{m}\left(0.5-\frac{\pi x^{2}}{2}\right)}=\frac{2 d z}{k_{m}\left(1-\pi\left(\epsilon^{2}-z^{2}\right)\right)}
$$

Upon combining Equations (25) to (23) and integrating,

$$
R_{2}=\frac{2}{k_{m}} \int_{0}^{\epsilon} \frac{\mathrm{d} z}{1+\pi(\kappa-1)\left(\epsilon^{2}-z^{2}\right)}=\frac{I_{u b}}{k_{m}}
$$

where

$$
I_{u b} \equiv 2 \int_{0}^{\epsilon} \frac{\mathrm{d} z}{1+\pi(\kappa-1)\left(\epsilon^{2}-z^{2}\right)}
$$

again has an analytical solution which is given by

$$
I_{u b}=\frac{2 \tanh ^{-1}\left[\epsilon \sqrt{\frac{\pi(\kappa-1)}{\pi(\kappa-1) \epsilon^{2}+1}}\right]}{\left.\sqrt{\pi(\kappa-1)\left[1+\pi(\kappa-1) \epsilon^{2}\right.}\right]}
$$

and $\kappa=k_{p} / k_{m}$ as before. The upper bound on the effective conductivity (non-dimensionalized with the conductivity of the surrounding medium) of the cell is thus given by

$$
\left(\frac{k_{e}}{k_{m}}\right)_{u b}=\left[1-2 \epsilon+2 I_{u b}\right]^{-1}
$$

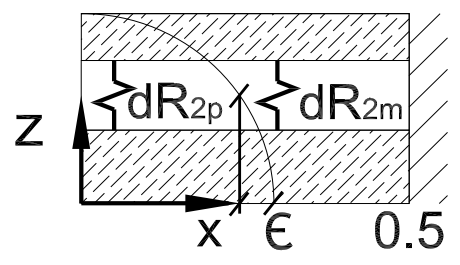

Figure $4 \quad R_{2}$ : The thermal resistance of the material inside a rectangular prism enclosing the particle

\section{Numerical Simulations}

Numerical simulations were performed in FEMLAB [14] to validate the model. Particle volume fractions $\left(\phi=V_{p} / V_{\text {cell }}\right)$ of $10,20,31,40$, and $52.4 \%$ and nondimensional particle conductivities $\left(\kappa=k_{p} / k_{m}\right)$ of 10 , 100 , and 1000 were studied. One quarter of the cell extending from one isothermal boundary to the other was selected as the computational domain. Approximately 150000 tetrahedral elements were used in each simulation and numerical results were shown to be within $1 \%$ of those from further refinement of the mesh.

The numerical work confirms that the upper and lower bounds are indeed valid (Figures 5 to 7 ). The geometric mean of the upper and lower bounds gives good agreement with the numerical results. The RMS error and maximum absolute difference between the two are expressed as percentages and are shown in Table 2. These are defined as follows:

$$
\mathrm{RMS} \equiv\left(\sqrt{\frac{\sum_{i=1}^{n} x_{i}^{2}}{n}}\right) \times 100 \%
$$

where

$x_{i} \equiv\left(\frac{\left(k_{e} / k_{m}\right)_{\text {model }}-\left(k_{e} / k_{m}\right)_{\text {numerical }}}{\left(k_{e} / k_{m}\right)_{\text {model }}}\right) \times 100 \%(33)$

and $\mathrm{n}$ is the number of points, in this case, 5 . The maximum absolute difference, $\left(x_{i}\right)_{\max }$, occurs in the vicinity of $\phi=40 \%$ for all three simulations. Both the RMS and the maximum absolute difference increase with $\kappa$.

The model consistently overpredicts the numerical results for the three cases studied. This suggests that the 3-D temperature field inside the cell more closely 
resembles that for one in which adiabats are assumed parallel to the direction of heat flow. Isotherms and lines of flux from the numerical results for $\kappa=100$ and $\phi=0.3$ are included in Figure 8 . The adiabats are neither parallel to the direction of heat flow (Figure 8 (a)) nor are the isotherms perpendicular to it (Figure 8 (b)). The temperature field is thus in some condition intermediate of the two.

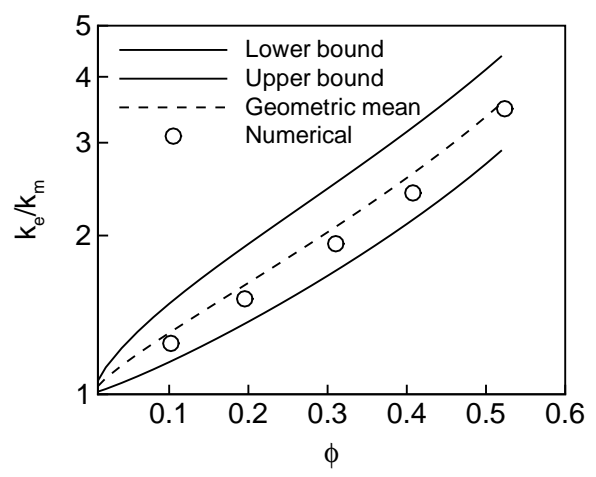

Figure 5 Numerical results versus upper and lower bounds for $\kappa=10$

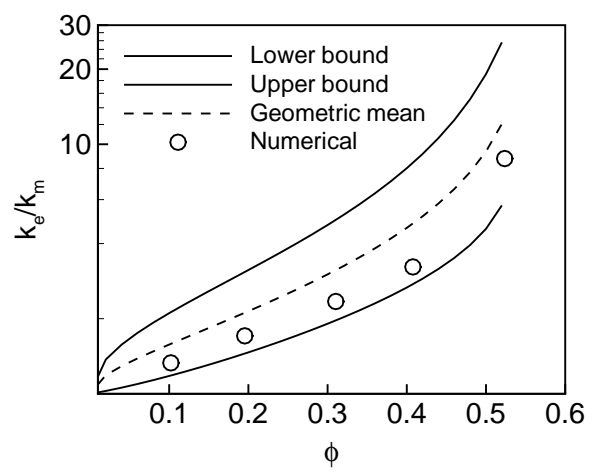

Figure 6 Numerical results versus upper and lower bounds for $\kappa=100$

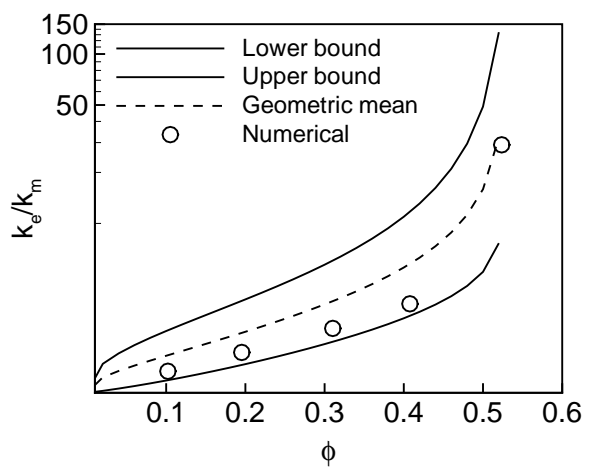

Figure 7 Numerical results versus upper and lower bounds for $\kappa=1000$

Karayacoubian et al., Thermal Resistance-Based Bounds...

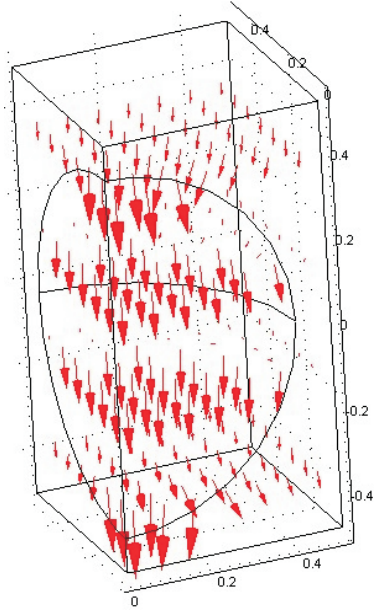

(a)

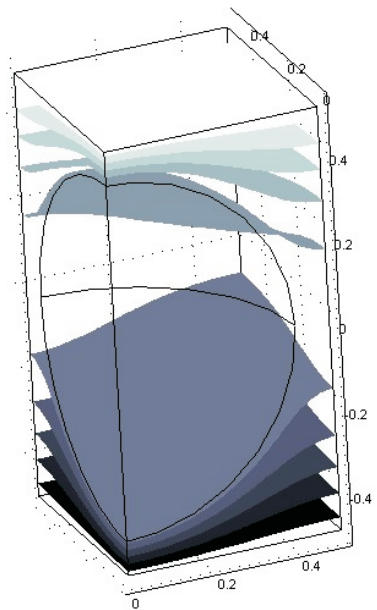

(b)
Figure 8 (a) Flux lines and (b) isotherms for $1 / 4$ cell $(\kappa=100, \phi=0.3)$

The effective conductivity of the cell for $\kappa=100$ and $\phi \leq 40 \%$ is within $4 \%$ of the effective conductivity of the cell for $\kappa=1000$. This confirms the result of many of the theoretical models (e.g. Maxwell [4]) which show that any increase in the particle conductivity beyond $\kappa \simeq 100$ produces very little further enhancement in the effective conductivity of the medium. In taking the limit of the upper and lower bounds in Equations (31) and (23),

$$
\begin{gathered}
\lim _{\kappa \rightarrow \infty}\left(\frac{k_{e}}{k_{m}}\right)_{u b}=(1-2 \epsilon)^{-1} \\
\lim _{\kappa \rightarrow \infty}\left(\frac{k_{e}}{k_{m}}\right)_{l b}=1-\pi \epsilon^{2}+2 \pi\left[\frac{1}{2} \ln \left(\frac{1}{1-2 \epsilon}\right)-\epsilon\right]
\end{gathered}
$$

The limiting enhancement is given by the geometric mean of Equations (34) and (35). For a typical particle volume fraction of $30 \%$, the particle conductivity must be increased by an order of magnitude (from $\kappa=100$ to 1000) for a further enhancement of just $10 \%$ in the effective conductivity. The effective conductivity for $\kappa=100$ is within approximately $30 \%$ of its maximum possible enhancement $(\kappa \rightarrow \infty)$ (Figure 9 ).

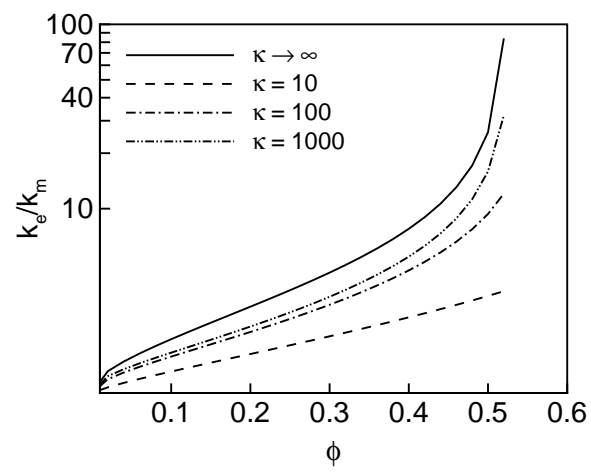

Figure 9 Limiting enhancement for $\kappa \rightarrow \infty$

22nd IEEE SEMI-THERM Symposium 
Table 2: RMS and maximum absolute difference between model and numerical results

\begin{tabular}{ccc}
$\kappa=k_{p} / k_{m}$ & RMS $(\%)$ & $\left(x_{i}\right)_{\max }(\%)$ \\
\hline 10 & 6.1 & 8.1 \\
100 & 25.8 & 33.0 \\
1000 & 30.6 & 41.9 \\
\hline
\end{tabular}

\section{Comparison with Experimental Data}

Experimental data of the effective thermal conductivity for composite systems were selected from the literature. Wong and Bollampaly [15] recently studied the enhancement in the conductivity of an epoxy resin by separately dispersing into it three different types of particles up to volume fractions of $\phi=50 \%$ (Figures 10 to 12$)$. The particles used were spherical silica $(\kappa=7.7)$, alumina $(\kappa=184.6)$, and silica-coated alumina nitride $(\kappa=1128.2)$. The samples were cured and solidified using a hardener. The agreement with the model for each case is very good. In particular, the model shows relatively good agreement for the alumina and silica-coated alumina nitride samples despite the irregular particle geometry.

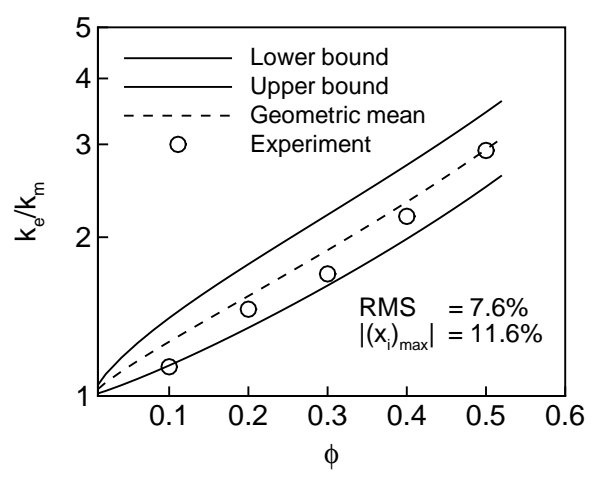

Figure 10 Experimental data from Wong and Bollampaly [15] for spherical silica particles $(\kappa=7.7)$ in an epoxy resin

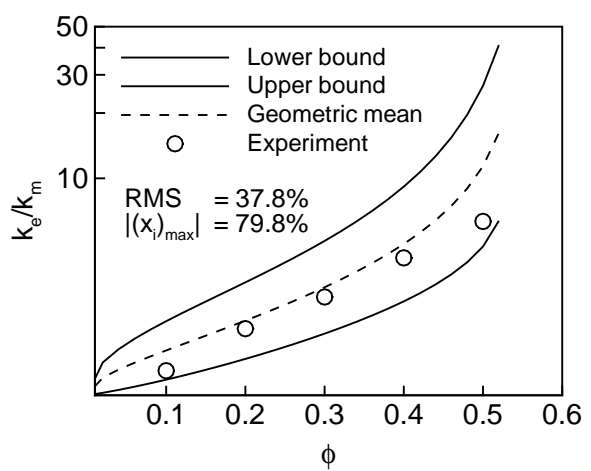

Figure 11 Experimental data from Wong and Bollampaly [15] for almost spherical alumina particles $(\kappa=184.6)$ in an epoxy resin

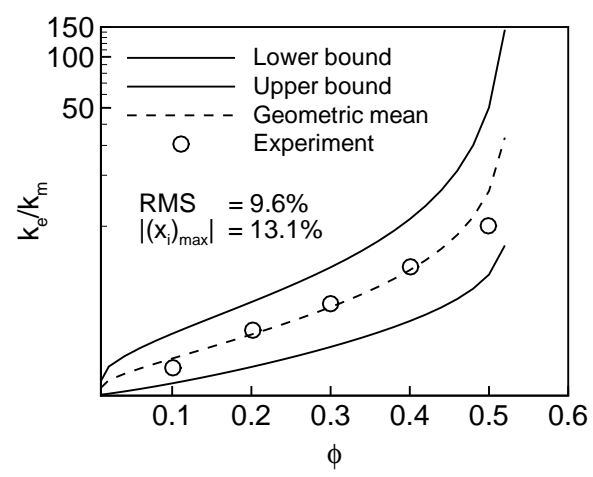

Figure 12 Experimental data from Wong and Bollampaly [15] for irregular SCAN particles $(\kappa=1128.2)$ in an epoxy resin

Sundstrom and Chen [16] studied the effective conductivity of glass dispersions in commercial polystyrene $(\kappa=7.3)$ for three different particle size ranges $(62-88$ $\mu \mathrm{m}, 125-149 \mu \mathrm{m}$, and 177-210 $\mu \mathrm{m}$ ) up to $\phi=40 \%$. The samples were melted under pressure in a mold and then solidified by cooling. The experimental results (Figure 13) show excellent agreement with the model giving an RMS error of $2.1 \%$ and maximum absolute difference less than $4 \%$.

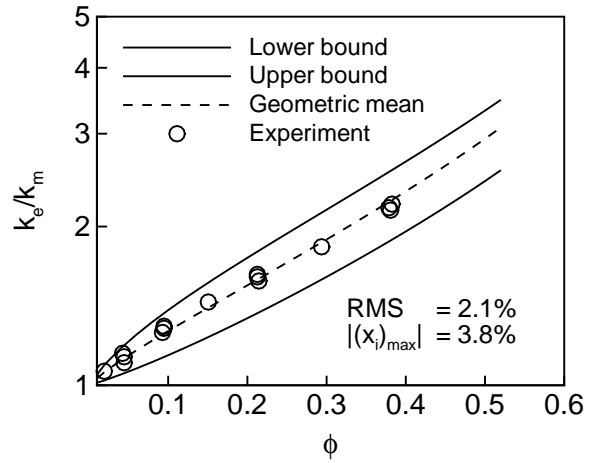

Figure 13 Experimental data from Sundstrom and Chen [16] for spherical glass particles in polystyrene $(\kappa=7.3)$

Tavman [17] studied a dispersion of aluminum oxide particles in commerical high density polyethylene $(\kappa=56.9)$. The polyethylene was in powder form and 
samples were prepared by the mold compression process. The average particle size was $10-20 \mu \mathrm{m}$. The model shows excellent agreement up to $\phi=10 \%$ beyond which the model begins to underpredict the experimental results (Figure 14). The maximum absolute difference is still $18 \%$, half of the maximum absolute error obtained from Maxwell's model.

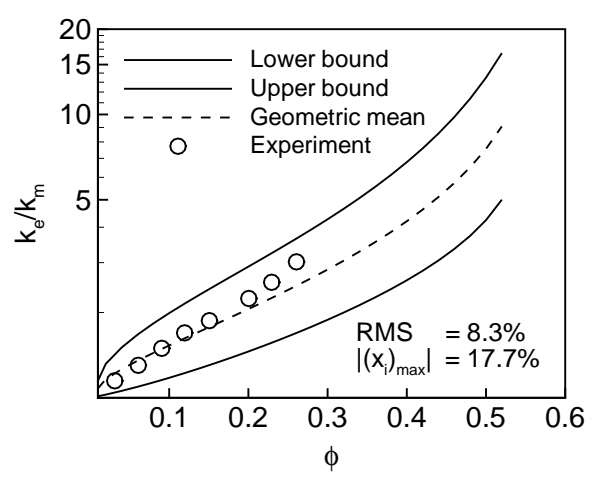

Figure 14 Experimental data from Tavman [17] for aluminum oxide particles in HDPE $(\kappa=56.9)$

Lin et al. [18] studied the effective conductivity of cupric oxide $(\kappa=1067.9)$ and aluminum powders $(\kappa=41.7)$ in an epoxy resin. The average particle size of the cupric oxide powder was $3 \mu \mathrm{m}$ and that of the aluminum powder, $7 \mu \mathrm{m}$. A transient method was used to determine the thermal diffusivity of cured spherical samples. The model shows excellent agreement for the composite of the cupric oxide powder (Figure 15). The RMS error is reasonable for the aluminum oxide powder composite $(9.4 \%)$ but it appears that any further loading of the sample will generate experimental results higher than the upper bound (Figure 16). The model falls $30 \%$ below the experimental measurement at $\phi=28 \%$ for this sample.

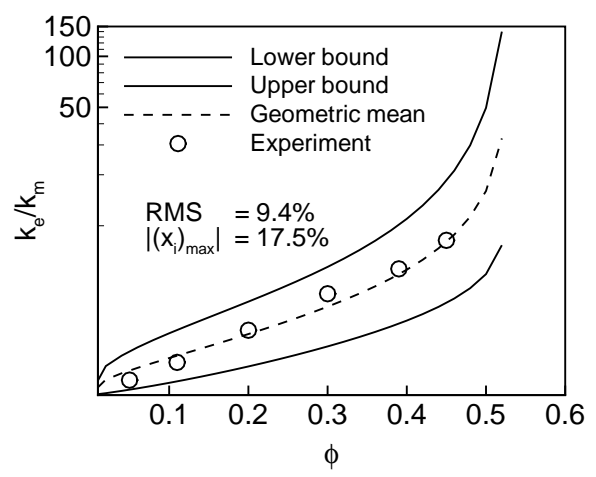

Figure 15 Experimental data from Lin et al. [18] for cupric oxide $(\kappa=1067.9)$ in an epoxy resin

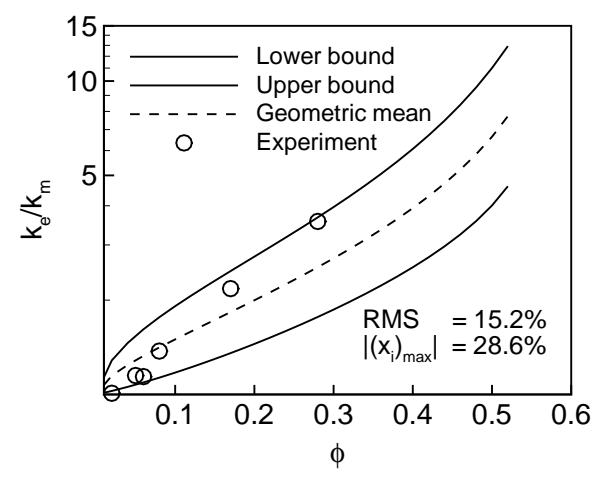

Figure 16 Experimental data from Lin et al. [19] for aluminum powders $(\kappa=41.7)$ in an epoxy resin

Woodside and Messmer [11] obtained experimental data for quartz sand packs surrounded by air $(\kappa=$ 325.8 ) for particle volume fractions of 41, 64, 69, and $81 \%$. The particle conductivity was assumed constant and equal for each sample $\left(k_{p}=8.4 \mathrm{~W} / \mathrm{mK}\right)$. The measurements were made using the transient hot wire method and found to agree well with other similar data. Recently, Carson et al. [19] showed that the data of Woodside and Messmer lie close to the lower bound of the series conduction model (Figure 17). The proposed model of a sphere in a unit cube is not able to capture the behaviour of these samples. A possible explanation of the unusual agreement of the experimental results with the series model is the settling of the quartz sand. In addition, note the relatively large volume fractions for which the data is obtained, alone an indicator of the potential failure of the model developed in this paper.

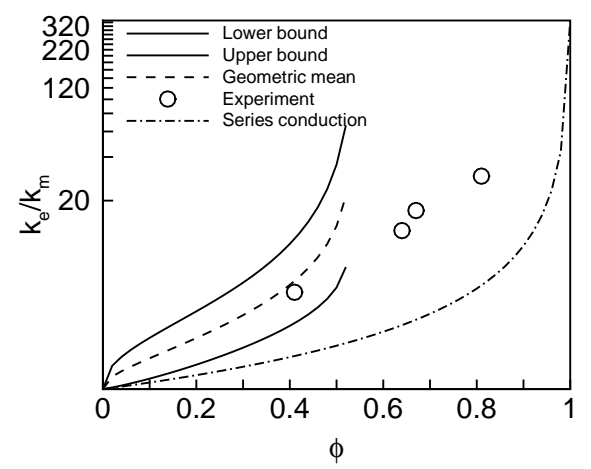

Figure 17 Experimental data from Woodside and Messmer [11] for quartz sand in air $(\kappa=325.8)$

Carson et al. [20] studied suspensions of expanded polystyrene (EPS) beads dispersed in a guar gel phase $(\kappa=19)$ for volume fractions from 40 to $100 \%$. A transient method based on the analytical solution for the temperature at the center of a sphere being cooled 
with convection boundary conditions was used. Experimental data were found to lie on the upper bound of the parallel conduction model (Figure 18). The gel was approximately 30 times more dense than the EPS beads. The parallel bound is applicable for systems in which the two phases are well-connected throughout along the axis of heat transfer. This suggests that the EPS beads formed continuous chain structures percolating from the center of the spherical sample to its outer boundary. Note again the high volume fractions of the dispersed phase achieved by the composite. In such sample, the sphere in a cube model presented in this paper cannot be applied because it is only valid up to $\phi \approx 52.4 \%$.

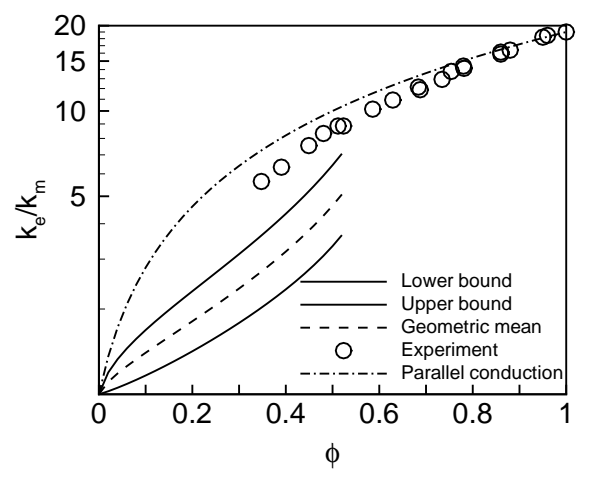

Figure 18 Experimental data from Carson et al. [20] for EPS beads in gel $(\kappa=19)$

\section{Summary and Conclusions}

The model developed in the present paper shows good agreement with numerical and experimental results. The model consistently overpredicts the numerical results for $\kappa=k_{p} / k_{m}$ of 10,100 , and 1000 . In the three cases studied, the RMS error and maximum absolute difference between the two increases with $\kappa$ and is largest for $\phi=40 \%$. The agreement with the selected experimental data is generally equally good. Although the model was developed for a spherical inclusion, the agreement with irregular geometries is still adequate for engineering purposes. There are limited instances when the dispersed phase in the composite is either well-connected forming continuous chains in the direction of heat flow or where the dispersed phase settles to a layer perpendicular to this direction. The model fails for these cases and the Weiner parallel and series conduction bounds become adequate models, respectively. Similar to effective medium theory, the current model indicates that any increase in $\kappa=k_{p} / k_{m}$ beyond $\sim 100$ for typical particle volume fractions results in very little further enhancement in the effective conductivity of the composite. For example, at $\phi=30 \%$ for a non-dimensional particle conductivity of $\kappa=100$,

Karayacoubian et al., Thermal Resistance-Based Bounds... the effective conductivity is within $30 \%$ of its maximum possible enhancement (for $\kappa \rightarrow \infty$ ). The model development is straightforward and the analysis can easily be performed and extended by engineers.

\section{Future Work}

Additional insight into the effective thermal conductivity of composites can be obtained if the present analysis is extended to dispersions of other geometries (ellipsoids, prismoids, right circular cylinders, etc.). A collection of such models for different particle geometries can help confirm whether the particle volume fraction is a suitable non-dimensional parameter. In addition, it can provide information regarding effects such as material distribution and particle orientation with respect to heat flow. The effects of packing could further be investigated in more detail if a characteristic cell of arbitrary dimensions is selected so that the particles are no longer constrained to lie at equal distances from one another in all directions.

\section{Acknowledgement}

The authors gratefully acknowledge the financial support of the Centre for Microelectronics Assembly and Packaging (CMAP) as well as the National Sciences and Engineering Research Council (NSERC). We are additionally indebted to the valuable conversations with postdoctoral fellow M. Bahrami of the University of Waterloo.

\section{References}

1. O. Weiner, 1912, Abh. Math. Phys. Kl. Konigl. Sach. Ges., 32, p. 509.

2. S. Torquato, 1991, "Random heterogeneous media: Microstructure and improved bounds on effective properties," Appl. Mech. Rev., 44(2), pp. 37-76.

3. G. W. Milton, 2002, Theory of Composites, Cambridge University Press, UK.

4. J. C. Maxwell, 1884, A Treatise on Electricity and Magnetism (3rd ed.), Dover Publications, Inc., USA, 9, p. 440.

5. A. Eucken, 1932, "Heat transfer in ceramic refractory materials (German)," Forsch. Geb. Ing. B3, 353(16), pp. 1-16.

6. R. L. Hamilton and O. K. Crosser, 1962, "Thermal conductivity of heterogeneous twocomponent systems," I \& EC Fund., 1(3), pp. 187-191.

7. Y. Benveniste, 1987, "Effective thermal conductivity of composites with a thermal contact resistance between the constituents: Nondilute case," J. Appl. Phys., 61, pp. 2840-2843.

22nd IEEE SEMI-THERM Symposium 
8. J. Felske, 2004, "Effective thermal conductivity of composite spheres in a continuous medium with contact resistance," Int. J. Heat Mass Transfer, 47, pp. 3453-3461.

9. R. C. McPhedran and D. R. McKenzie, 1978, "The conductivity of lattices of spheres. I. The simple cubic lattice," Proc. Royal Soc. London Ser. A, 359(1696), pp. 45-63.

10. D. A. G. Bruggeman, 1935, "Calculation of various physical constants in heterogeneous substances. I. Dielectric constants and conductivity of composites from isotropic substances (German)," Annalen der Physics, 24(5), pp. 636-679.

11. W. Woodside and J. H. Messmer, 1961, "Thermal conductivity of porous media. I. Unconsolidated sands," J. Appl. Phys., 32(9), pp. 1688-1699.

12. S. C. Cheng, R. I. Vachon, 1969, "The prediction of thermal conductivity of two and three phase solid heterogeneous mixtures," Int. J. Heat Mass Transfer, 12, pp. 249-264.

13. T. B. Lewis and L. E. Nielsen, 1970, "Dynamic mechanical properties of particulate-filled oolymers," J. Appl. Polym. Sci., 14, pp. 1449-1471.

14. FEMLAB 3.0a, 3.0.0.228, Copyright (c) by COMSOL AB.
15. C. P. Wong and R. S. Bollampally, 1999, "Thermal conductivity, elastic modulus, and coefficient of thermal expansion of polymer composites filled with ceramic particles for electronic packaging," J. Appl. Polymer Sci., 74, pp. 3396-3403.

16. D. W. Sundstrom and S. Y. Chen, 1970, "Thermal conductivity of reinforced plastics," J. Comp. Mat., 4, pp. 113-117.

17. I. H. Tavman, 2000, "Thermal conductivity of particle reinforced polymer composites," Int. Comm. Heat Mass Transfer, 27(2), pp. 253-261.

18. F. Lin, G. S. Bhatia, and J. D. Ford, 1993, "Thermal conductivities of powder-filled epoxy resins," J. Appl. Polymer Sci., 49, pp. 1901-1908.

19. J. K. Carson, S. J. Lovatt, D. J. Tanner, and A. C. Cleland, 2005, "Thermal conductivity bounds for isotropic, porous materials," Int. J. Heat Mass Transfer, 48, pp. 2150-2158.

20. J. K. Carson, S. J. Lovatt, D. J. Tanner, and A. C. Cleland, 2004, "Experimental measurements of the effective thermal conductivity of a pseudoporous food analogue over a range of porosities and mean pore sizes," J. Food Eng., 63, pp. 8795. 